In der Rubrik „Literatur kompakt" werden die wichtigsten Originalarbeiten aus der internationalen Fachliteratur referiert.

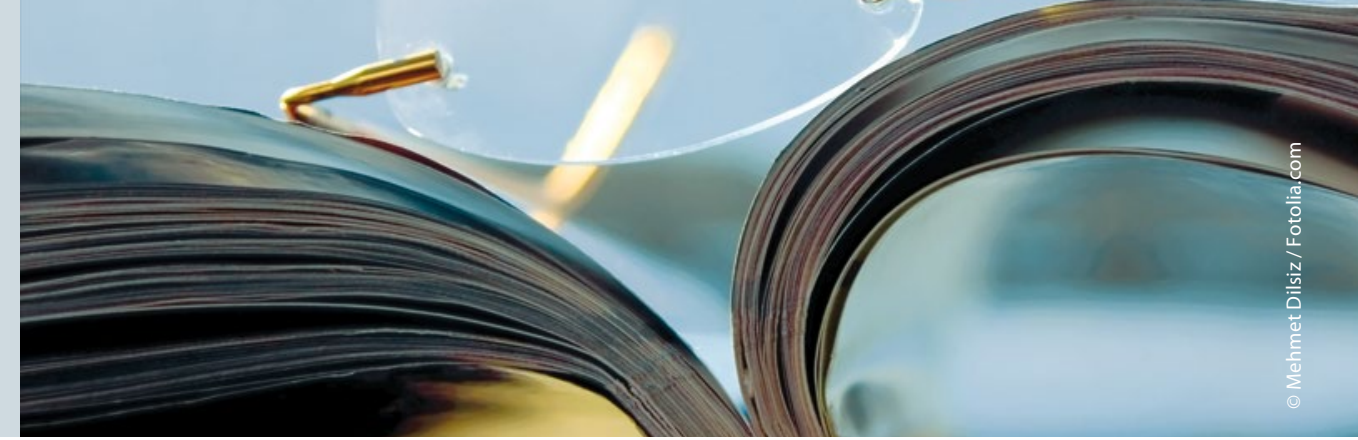

\section{Adipositas: Abspecken vor Herz-OP unnötig?}

\section{Die Adipositas erhöht das Risiko für Herz-Kreislauf-Erkrankungen und die Sterblichkeit. Bei Herzoperationen haben Adipöse jedoch einen Vorteil, wie aktuelle Daten zeigen.}

Cit 1983 ist bekannt, dass die Adipositas die Entwicklung von Hypertonie, korornarer Herzkrankheit und Herzinsuffizienz begünstigt. In den letzten Jahren gab es jedoch einige Publikationen, die zeigten, dass adipöse Patienten im Vergleich zu Normalgewichtigen einen gesundheitlichen Vorteil haben, wenn Erkrankungen wie eine koronare Herzerkrankung, eine Herzinsuffizienz oder eine terminale Niereninsuffizienz bereits existent sind und eine Herzoperation bei ihnen durchgeführt wird. Das Phänomen wird Adipositas-Paradoxon genannt.

Nun liegen zu Herzoperationen bei adipösen Patienten Daten aus einer Metaanalyse mit 26 Studien und einer Kohortenstudie mit prospektiv erhobenen Daten von 557.720 in den Jahren 2002 bis 2013 Operierten aus Großbritannien/ Irland vor. In beiden Studien zeigte sich eine U-förmige Assoziation zwischen der Mortalität und dem Body-Mass-Index (BMI). In der Kohorte war das relative Mortalitätsrisiko für Übergewichtige (BMI 25-29,9 kg/m²) um $21 \%$ und für Adipöse (BMI $30-40 \mathrm{~kg} / \mathrm{m}^{2}$ ) um $19 \% \mathrm{im}$ Vergleich zu Normalgewichtigen (BMI $18,5-24,9 \mathrm{~kg} / \mathrm{m}^{2}$ ) vermindert und für Untergewichtige $\left(\mathrm{BMI}<18,5 \mathrm{~kg} / \mathrm{m}^{2}\right) \mathrm{um}$ $51 \%$ erhöht. Ähnliche Ergebnisse zeigten sich in der Metaanalyse.

Vorteile hatten Adipöse auch dann, wenn eine reduzierte Ejektionsfraktion $(<30 \%)$ vorlag oder ein Zustand nach Schlaganfall bestand, nicht jedoch bei bestehender Niereninsuffizienz/Dialyse. Den größten Vorteil hatten Adipöse, die sich einer Bypassoperation unterzogen.

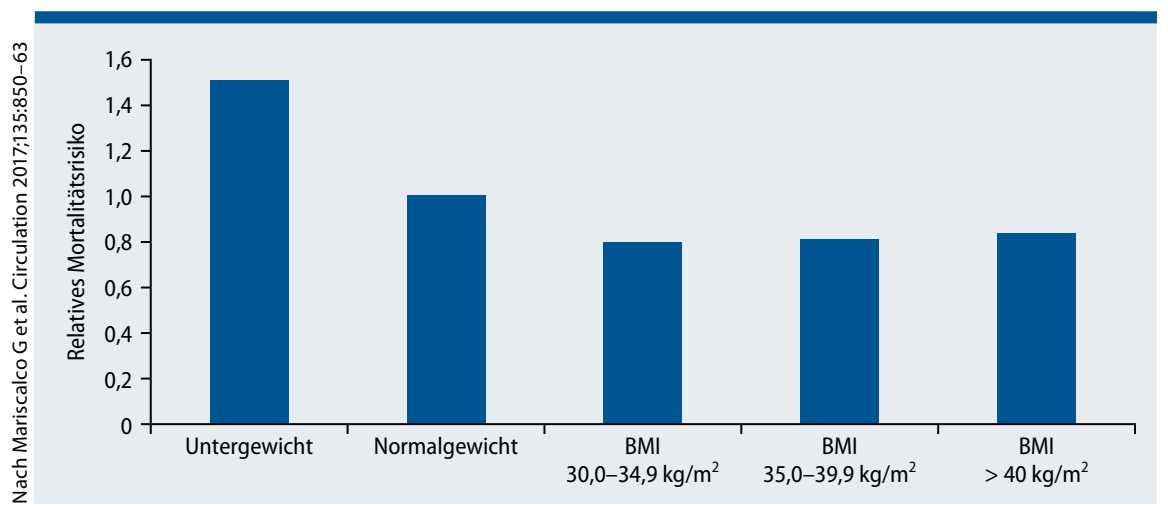

Abb. 1: Relatives Mortalitätsrisiko bei Herzoperationen in Abhängigkeit vom Körpergewicht.

Sternale Wundinfektionen waren jedoch bei Adipösen häufig festzustellen. Untergewicht stellte sich bei allen untersuchten Parametern als erhebliches Mortalitätsrisiko heraus.

Mariscalco $\mathrm{G}$ et al. Body mass index and mortality among adults undergoing cardiac surgery. A nationwide study with a systematic review and meta-analysis. Circulation. 2017;135:850-63

\section{Kommentar}

Die vorliegende Studie mit zwei großen Kollektiven (Kohorte und Metaanalyse) bestätigt und relativiert aufgrund der wissenschaftlichen Aussagekraft frühere Untersuchungen. Mit Ausnahme von Patienten mit Niereninsuffizienz hatte eine Adipositas generell Vorteile, was die klinische Beurteilung verändern wird. Bisher wird in nationalen und internationalen Leitlinien bei Adipösen eine Gewichtsreduktion vor einer Herzoperation empfohlen, eine womöglich nicht mehr lang bestehende Empfehlung. Die Mechanismen, die den Überlebensvorteil von Adipösen bei Herzoperationen erklären, sind noch nicht bekannt. In früheren Studien waren Adipöse häufig jünger, hatten eine bessere Herzfunktion und erhielten mehr Pharmaka. In der Kohorte war das Alter in den BMI-Gruppen ebenfalls unterschiedlich: Normalgewichtige waren im Mittel 69 Jahre, Adipöse mit einem BMI von $35-39,9 \mathrm{~kg} / \mathrm{m}^{2} 64$ Jahre und mit einem BMI $>40 \mathrm{~kg} / \mathrm{m}^{2} 62$ Jahre alt.

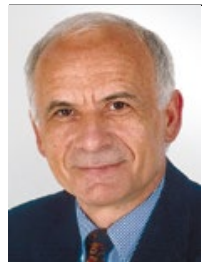

Prof. Dr. med. Alfred Wirth Bad Rothenfelde 